\title{
A CASE REPORT OF SPONTANEOUS CORONARY ARTERY DISSECTION
}

\author{
Yusuf Can Özdemir ${ }^{1}$, Kubilay Elmacı ${ }^{1}$, Fatih Özçelik ${ }^{2}$
}

${ }^{1}$ Trakya University School of Medicine, Edirne, TURKEY

${ }^{2}$ Department of Cardiology, Trakya University School of Medicine, Edirne, TURKEY

\section{ABSTRACT}

Aims: Spontaneous coronary artery dissection is a random rupture of the coronary artery wall, which may lead to ischemia of the heart tissue. $30 \%$ of the cases are male patients. The aim of this article is to report a patient with spontaneous coronary artery dissection, raise awareness and extend the literature.

Case Report: A 43-year-old male patient was presented to Trakya University Hospital with a complaint of persistent chest pain. The patient underwent coronary angiography, revealing spontaneous coronary artery dissection in the left anterior descending artery and circumflex artery.

Conclusion: In this case report, we demonstrated the diagnosis of spontaneous coronary artery dissection with coronary angiography. Our patient had the most common dissection, left anterior descending artery, right coronary artery and left main coronary artery. Only medical therapy could be started for the patient due to his voluntary discharge.

Keywords: Coronary vessels, coronary artery disease, coronary angiography, chest pain

\section{INTRODUCTION}

Spontaneous coronary artery dissection (SCAD) is a random rupture of the coronary artery wall. SCAD is a rare defect, mostly affecting women. Especially in peripartum period, it can be fatal (1-3). The severity of SCAD is determined by obstruction ratio of blood flow and the diagnosis is generally made with coronary angiography (CAG) (4). Currently, management of SCAD is largely based on expert opinions (5). Medications may be administered to prevent coagulation and to reduce chest pain, blood pressure, cholesterol. Another treatment method for SCAD is percutaneous coronary intervention.

Spontaneous coronary artery dissection is not a newly discovered case. It was first reported by Pretty (1931) after the unexpected death of a patient who had symptoms such as vomiting, indicating coronary artery rupture (6). With the advances in imaging and diagnostic techniques, awareness of SCAD has raised greatly (7).

There is not consensus on categorizing SCAD. Traditionally, patients with SCAD are studied under four groups: patients with congenital connective tissue disorders that cause arterial wall defects, idiopathic SCAD, women in the peripartum period and patients suffering from atherosclerosis, particularly men at the age of 55 on average (8). However, on the contrary, modern definition of SCAD excludes patients with atherosclerosis (5).

There are two possible ways for the development of intramural hematoma with SCAD. The first one is intimal rupture causing blood flow into the intimal space, generating a false lumen within the artery; the second one is rupture of the vasa vasorum (9).

Three different monitoring methods can be used to diagnose SCAD. The first one is coronary angiography which provides a two-dimensional image limits the identification of the type of luminal obstruction (10). The second way is optical coherence tomography and intravascular ultrasound that gives information about the condition of the artery wall. Compared to CAG that only shows the lumen, which can be of advantage (11). The third way is cardiac computed tomography that is not common to evaluate SCAD due to its lower resolution (11). This method can only be used if the dissection is formed in a proximal segment of the artery (12). 
The aim of this article is to report a patient with spontaneous coronary artery dissection, raise awareness and extend the literature.

\section{CASE REPORT}

A 43-year-old male patient presented to an outside facility complaining of chest pain, then was referred to Trakya University Hospital after his cardiac stress test results were found significant. The patient had a history of urinary tract infection; however a history of diabetes mellitus, hypertension, old myocardial infarction or coronary artery disease were not present. Continuous usage of tobacco and alcohol was noted. The drugs used by our patient were acetylsalicylic acid (ASA) and beta blockers. His blood pressure was $135 \mathrm{mmHg}$ systolic and $70 \mathrm{mmHg}$ diastolic on admission. His electrocardiogram (ECG) had negative T waves in V5-V6 (Figure 1). Physical examinations were normal in this case. Echocardiography that was performed before CAG revealed the following results: systolic dysfunction, global hypokinesia, ejection fraction deficiency, concentric hypertrophy and type 1 diastolic dysfunction in the left ventricle; mild mitral and aortal insufficiencies. An appointment was scheduled for coronary angiography and routine tests.

The patient was hospitalized in the cardiology unit one day before CAG and his usual medication was provided. Routine tests showed high platelet distribution width, high number of basophils, high levels of LDL-C, ALT, CRP, Ig-G, prothrombin time and low mean platelet volume and sodium levels.
Coronary angiography revealed spontaneous spiral diffuse dissection of circumflex artery (CX), starting from the proximal part and extending to the middle, dissection of the left anterior descending artery (LAD), starting from the proximal part and extending to S2 (Figure 2). Distal Thrombolysis in Myocardial Infarction (TIMI) 3 flow was detected in both CX and LAD. In ventriculography, anterior apical and inferior hypokinesia was found. The board diagnosed the case as coronary artery disease. Myocardial perfusion scanning (MPS) was considered necessary and scheduled.

Spironolactone, clopidogrel, perindopril, beta blockers and acetylsalicylic acid were given until his discharge. As a result of this medication regimen, angina disappeared and his systolic blood pressure decreased to $110 \mathrm{mmHg}$. While the patient was still being monitored in the cardiology service, another CAG was performed to check if any further operation is needed.

The second CAG revealed a dissection and $70 \%$ stenosis after the first diagonal branch (D1) and 70\% stenosis in the ostium of D1; also, a $5 \mathrm{~cm}$ spiral dissection starting from proximal and extending to the distal part of CX. For the treatment decision (medication, stent implantation or coronary artery bypass grafting), the board decided to evaulate the MPS results.

The patient wanted to be discharged even when he was informed about all the risks of his condition before MPS was done. Perindopril, clopidogrel, ASA, atorvastatin, pantoprazole and metoprolol were given to the patient for regular use after his discharge.
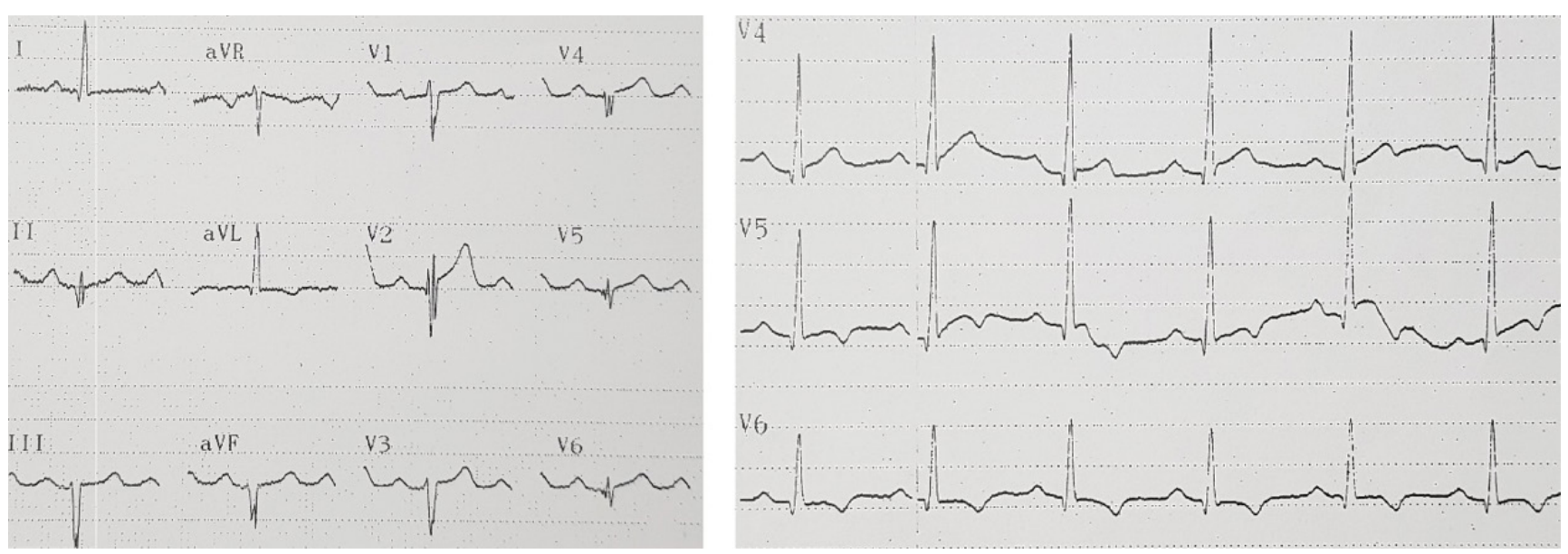

Figure 1: ECG findings of the patient, revealing negative T wave in V5-V6. 

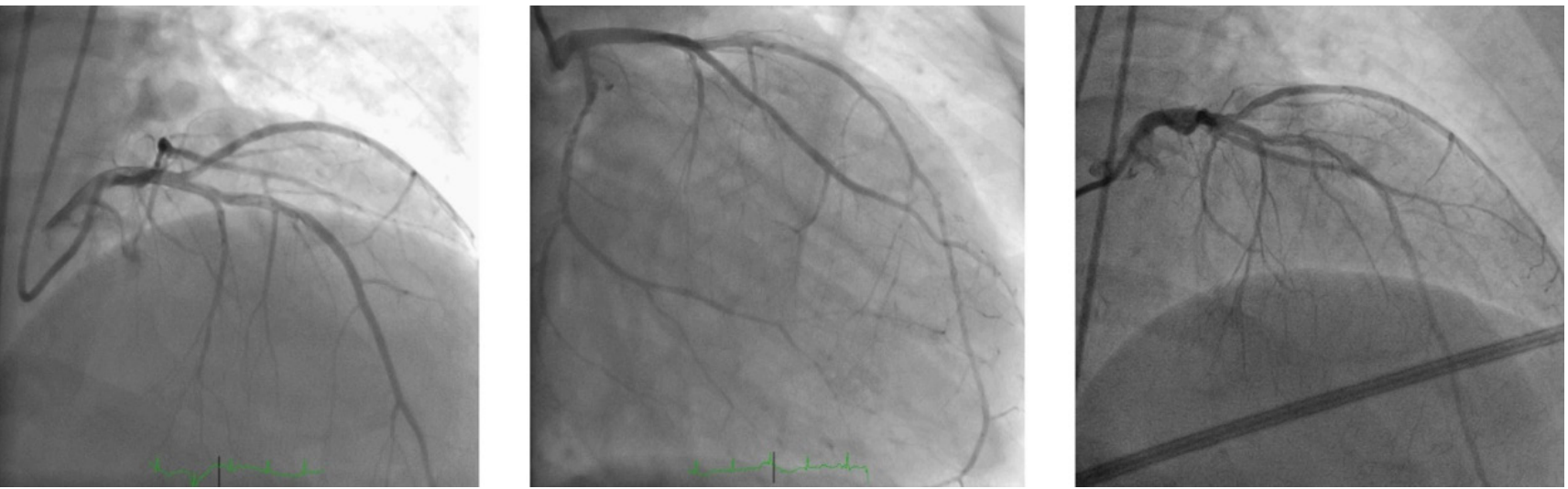

Figure 2: CAG findings of the patient, revealing dissections of LAD and CX, normal right coronary artery.

\section{DISCUSSION}

Gender distribution for SCAD is unbalanced. 30\% of the SCAD patients are male, as in our case $(13,14)$. There were no risk factors present for our patient, apart from an intensive use of tobacco and alcohol. The most common three dissection areas are LAD, right coronary artery and left main artery, respectively (15). Our patient had a dissection of $\mathrm{LAD}$, which is the most frequently involved coronary artery.

Depending on the severity of ischemia, culmination in acute coronary syndrome (ACS) and clinical findings, coronary reperfusion can be provided with stent implantation in spontaneaus dissections. Coronary artery bypass grafting can be offered to the patients with coronary anatomies that are unsuitable for stent implantation or in clinically stable patients without angina and ACS, medical therapy can be a choice.

In the management of SCAD, medical therapy plays an important role. Medication includes beta-blockers, angiotensin-receptor blockers, anticoagulants, thrombolytic therapies, antiplatelet therapies and lipid-lowering therapies (5). As our patient was clinically stable and did not have angina or ACS, the decision for a MPS analysis was made in order to determine if the dissections resulted in a serious ischemia. In our case, medical therapy was the only treatment as the patient denied MPS analysis and treatment, and wanted to be discharged, preventing us from making the analysis and completing the treatment.

To conclude, CAG is the current gold standard to diagnose SCAD. According to size and the type of the dissection, appropriate treatment should be determined by specialists.
Ethics Committee Approval: N/A

Informed Consent: Written informed consent was obtained from the participants of this study.

Conflict of Interest: The authors declared no conflict of interest.

Author contributions: Concept: FÖ, YCÖ, KE. Design: FÖ, YCÖ, KE. Supervision: FÖ. Resources: FÖ, YCÖ, KE. Materials: FÖ, YCÖ, KE. Data collection and/or processing: FFÖ, YCÖ, KE. Analysis and/or Interpretation: FÖ, YCÖ, KE. Literature Search: FÖ, YCÖ, KE. Writing Manuscript: FÖ, YCÖ, KE. Critical Review: FÖ.

Financial disclosure: The authors declared that this study received no financial support.

Editor-in-chief's Note: One of the authors of this article, Kubilay Elmac1 is a member of the editorial board of Turkish Medical Student Journal. However, he did not take place in any stage on the editorial decision of the manuscript. The editors who evaluated this manuscript are from another institutions.

\section{REFERENCES}

1. Tweet MS, Hayes SH, Pitta SR et al. Clinical feature, management and prognosis of spontaneous coronary artery dissection. Circulation 2012;126:579-88.

2. Adlam D, Cuculi F, Lim C et al. Management of spontaneous coronary artery dissection in the primary percutaneous coronary intervention era. J Invasive Cardiol 2010;22:549-53.

3. Jorgensen MB, Aharonian V, Mansukhani $\mathrm{P}$ et al. Spontaneous coronary dissection: a cluster of cases with this rare finding. Am Heart J 1994;127:1382-7. 
4. Mokhberi V, Bagheri B, Navidi S et al. Spontaneous coronary artery dissection: a case report. J Teh Univ Heart Ctr 2015;10(3):159-62.

5. Saw J, Mancini J, Humphries HK. Contemporary review on spontaneous coronary artery dissection. JACC 2016;68(3):297-312.

6. Pretty HC. Dissecting aneurysm of coronary artery in a woman aged 42: rupture. BMJ 1931;1:667.

7. Tweet MS, Gulati R, Aase LA et al. Spontaneous coronary artery dissection: a disease-specific, social networking community-initiated study. Mayo Clin Proc 2011;86:845-50.

8. Sabatine MS, Jaffer FA, Staats PN et al. Case records of the massachusetts general hospital. Case 28-2010. A 32-year-old woman, 3 weeks post partum, with substernal chest pain. N Engl J Med 2010;363:1164-73.

9. Yip A, Saw J. Spontaneous coronary artery dissection-a review. Cardiovasc Diagn Ther 2015;5(1):37-48.

10. Al Emam AR, Almomani A, Gilani SA et al. Spontaneous coronary artery dissection: one disease, variable presentations, and different management approaches. International Journal of Angiography 2016;25(3):13947.

11. Saw J. Coronary angiogram classification of spontaneous coronary artery dissection. Catheter Cardiovasc Interv 2014;84(7):1115-22.

12. Wain-Hobson J, Roule V, Dahdouh Z et al. Spontaneous coronary artery dissection: one entity with several therapeutic options. Cardiovasc Revasc Med 2012;13(3):203.

13. DeMaio SJ Jr, Kinsella SH, Silverman ME. Clinical course and long-term prognosis of spontaneous coronary artery dissection. Am J Cardiol 1989;64:471-4.

14. Thompson EA, Ferraris S, Gress T et al. Gender differences and predictors of mortality in spontaneous coronary artery dissection: a review of reported cases. J Invasive Cardiol 2005;17:59-61.

15. Zampieri P, Aggio S, Roncon L et al. Follow up after spontaneous coronary artery dissection: a report of five cases. Heart 1996;75:206-9. 